\title{
ABCA1 overexpression leads to hyperalphalipoproteinemia and increased biliary cholesterol excretion in transgenic mice
}

\author{
Boris L. Vaisman, ${ }^{1}$ Gilles Lambert, ${ }^{1}$ Marcelo Amar, ${ }^{1}$ Charles Joyce, ${ }^{1}$ Toshimitsu Ito, ${ }^{1}$ \\ Robert D. Shamburek, ${ }^{1}$ William J. Cain, ${ }^{2}$ Jamila Fruchart-Najib, ${ }^{3}$ Edward D. Neufeld, ${ }^{1}$ \\ Alan T. Remaley, ${ }^{1}$ H. Bryan Brewer, Jr., ${ }^{1}$ and Silvia Santamarina-Fojo ${ }^{1}$ \\ ${ }^{1}$ Molecular Disease Branch, National Heart, Lung, and Blood Institute, Bethesda, Maryland, USA \\ ${ }^{2}$ Biological Sciences, University of Delaware, Newark, Delaware, USA \\ ${ }^{3}$ Department d'Atherosclerosis, Pasteur Institute, Lille, France \\ Address correspondence to: Boris L. Vaisman, Molecular Disease Branch, NHLBI-NIH, Building 10, Room 7N115, \\ 10 Center Drive, Bethesda, Maryland 20892-1666, USA. \\ Phone: (301) 496-3210; Fax: (301) 402-0190; E-mail: boris@mail.nih.gov. \\ Boris L. Vaisman and Gilles Lambert contributed equally to this work. \\ Received for publication February 14, 2001, and accepted in revised form June 12, 2001.
}

The discovery of the ABCA1 lipid transporter has generated interest in modulating human plasma HDL levels and atherogenic risk by enhancing ABCA1 gene expression. To determine if increased ABCA1 expression modulates HDL metabolism in vivo, we generated transgenic mice that overexpress human ABCA1 (hABCA1-Tg). Hepatic and macrophage expression of hABCA1 enhanced macrophage cholesterol efflux to apoA-I; increased plasma cholesterol, cholesteryl esters (CEs), free cholesterol, phospholipids, HDL cholesterol, and apoA-I and apoB levels; and led to the accumulation of apoErich HDL1. ABCA1 transgene expression delayed ${ }^{125} \mathrm{I}$-apoA-I catabolism in both liver and kidney, leading to increased plasma apoA-I levels, but had no effect on apoB secretion after infusion of Triton WR1339. Although the plasma clearance of HDL-CE was not significantly altered in hABCA1-Tg mice, the net hepatic delivery of exogenous $3 \mathrm{H}-\mathrm{CEt}-\mathrm{HDL}$, which is dependent on the HDL pool size, was increased 1.5-fold. In addition, the cholesterol and phospholipid concentrations in hABCA1-Tg bile were increased 1.8-fold. These studies show that steady-state overexpression of ABCA1 in vivo (a) raises plasma apoB levels without altering apoB secretion and (b) raises plasma HDL-C and apoA-I levels, facilitating hepatic reverse cholesterol transport and biliary cholesterol excretion. Similar metabolic changes may modify atherogenic risk in humans.

J. Clin. Invest. 108:303-309 (2001). DOI:10.1172/JCI200112517.

\section{Introduction}

In humans, the risk of coronary artery disease is inversely correlated to the plasma levels of HDLs (1). One of the major processes by which HDL may function as an antiatherogenic lipoprotein is by facilitating the transfer of cholesterol from peripheral cells to the liver for biliary excretion (2). Oram and Yokoyama (3) first proposed that cell-surface proteins might be involved in the efflux of cellular cholesterol to HDL. Recently, the ATPbinding cassette transporter 1 (ABCA1) has been identified as the key transporter that facilitates this initial step in reverse cholesterol transport.

ABCA 1 is a member of a large family of evolutionarily conserved transmembrane proteins that transport a wide variety of molecules, including proteins, lipids, ions, and sugars across membranes $(4,5)$. The entire genomic sequence of the human ABCA1 gene has been recently reported $(6,7)$. It spans a total of $149 \mathrm{~kb}$ that consists of 50 exons (7-9) and encodes a protein that contains 2,261 amino acids $(7,9)$. The transporter is widely expressed in different tissues such as the liver, placenta, lung, adrenals, and macrophages (10). Its expres- sion is highly regulated by cAMP and sterols (10-14). Mutations in ABCA1 lead to Tangier disease and familial hypoalphalipoproteinemia $(6,11,15-19)$, genetic disorders characterized by marked reductions in HDL plasma levels and increased risk of cardiovascular disease.

ABCA1 mediates the active removal of cellular cholesterol and phospholipids to lipid-poor apolipoproteins from a variety of cells $(14,20,21)$. ABCA1-dependent lipid efflux from cells represents the initial step in the process of reverse cholesterol transport (2), one of the major mechanisms by which HDL may function as an antiatherogenic lipoprotein. Thus, increased ABCA1 expression would be anticipated to enhance reverse cholesterol transport and reduce atherogenic risk. ABCA1 has been identified as a potential target to develop new pharmacological agents that may raise plasma HDL-cholesterol levels in humans. ABCA1 deficiency in humans $(8,11,15-19)$ and mice $(22-24)$ leads to decreased plasma HDL levels. However, at present it is not known whether increased expression of ABCA1 will raise plasma HDL concentrations or enhance biliary cholesterol excretion. 
To gain a better understanding of ABCA1 function and determine the consequences of enhanced $\mathrm{ABCA} 1$ gene expression on lipoprotein metabolism, we have produced two lines of transgenic mice that overexpress the human ABCA1 gene in liver and macrophages. In this study, we report the effect of human ABCA1 overexpression on the plasma lipid profile, HDL metabolism, bile acid composition, and reverse cholesterol transport in transgenic mice.

\section{Methods}

Generation of human $A B C A 1$ transgenic mice. The fulllength (6.78-kb) human ABCA1 cDNA (7) was cloned into the expression plasmid pLIV.11 (25) modified by the addition of NotI linkers. After digestion with SalI and SpeI, a $16.7-\mathrm{kb}$ DNA fragment containing the human apoE promoter, the human $\mathrm{ABCA} 1 \mathrm{CDNA}$, the polyA signal from the human apoE gene, and the hepatic and macrophage control regions of the apoE/apoC-I locus $(7,25)$ was isolated and injected into the male pronucleus of fertilized eggs from superovulated C57BL/6N females (Charles River Laboratories, Wilmington, Massachusetts, USA). Integration of human ABCA1 cDNA in newborn mice was determined by dot blot and Southern blot hybridization of genomic mouse tail DNA using full-length hABCA1 cDNA as probe. The mice were housed under protocols approved by the Animal Care and Use Committee of the National Heart, Lung, and Blood Institute.

Northern and Western blot analysis. Northern blotting was performed using 20-30 $\mu \mathrm{g}$ of total RNA isolated from control $(n=3)$ and hABCA1-Tg $(n=3)$ liver, brain, heart, kidney, lung, spleen, adrenals, skeletal muscle, and small intestine as well as unstimulated pooled $(n=15)$ peritoneal macrophages. The human ABCA1 (6.78 kb), mdr2 (679 bp), and $\beta$-actin (1.076 $\mathrm{kb})$ (DECAtemplate $\beta$-actin mouse; Ambion Inc., Austin, Texas, USA) cDNAs were used as hybridization probes. Densitometric scanning was performed using the Molecular Dynamics Personal Densitometer SI (Sunnyvale, California, USA). Western blot analysis of mouse liver and macrophage homogenates $(25 \mu \mathrm{g})$ was performed using either polyclonal anti-mouse LRP (Research Diagnostics Inc., Flanders, New Jersey, USA), LDLr-IgG (RDI), SR-BI (Novus, Littleton, Colorado, USA) antibodies or an anti-human ABCAI antibody isolated from chicken eggs after immunization with a 41 amino acid synthetic peptide (FFIRPRPVNAKLSPLNDEDEDVRRERQRILDGGDQNDILEI), corresponding to a region near the carboxy terminus of the human ABCAI protein.

Plasma lipid, lipoprotein, and apolipoprotein analysis. Male and female control and hABCA1-Tg mice (2-3 months of age) were maintained on a regular chow $\operatorname{diet}(\mathrm{NIH}-$ 31 chow diet; Zeigler Bros. Inc., Gardners, Pennsylvania, USA). The plasma cholesterol (TC), triglycerides (TGs), phospholipids (PLs), free cholesterol (FC), and HDL-C after a 4-hour fast were quantified as described elsewhere (26). The apoA-I, apoA-II, apoE, and apoB levels of hABC1-Tg and control mice plasma samples $(0.1 \mu \mathrm{l})$ and FPLC fractions $(10 \mu \mathrm{l})$ were analyzed by Western blot using antibodies from Biodesign International (Saco, Maine, USA). Mouse apolipoproteins A-I, A-II, E, and B were quantified by ELISA (27). Plasma lipoproteins were analyzed by gel filtration on two Superose 6 columns in series (FPLC; Amersham Pharmacia Biotech, Piscataway, New Jersey, USA). Twodimensional gel electrophoresis of plasma lipoproteins was performed as described previously (28).

Efflux studies. Cholesterol efflux to apoA-I and HDL was measured in quintuplicate in two independent experiments as described elsewhere (21) after incubating peritoneal macrophages for 24 hours in DMEM (1 $\mathrm{mg} / \mathrm{ml} \mathrm{BSA}, 10 \mu \mathrm{g} / \mathrm{mL}$ acetylated LDL, and $1 \mu \mathrm{Ci} / \mathrm{ml}$ ${ }^{3} \mathrm{H}$-cholesterol). Data were normalized to cell protein and expressed as percent of total counts.

HDL metabolic studies. Mouse HDL labeled with ${ }^{3} \mathrm{H}-$ cholesteryl-palmityl-ether (CEt) (29), ${ }^{125}$ I-tyramine-cellobiose-labeled (TC-labeled) mouse-apoA-I (30), and ${ }^{125}$ I-labeled apoA-I (31) were prepared. Each radiolabeled apolipoprotein $(50 \mu \mathrm{g}$ apoA-I) was separately mixed with $200 \mu \mathrm{l}$ of control mouse plasma. ${ }^{125}$ I-tyramine-cellobiose-apoA-I-labeled mouse HDL was further separated by FPLC. After extensive dialysis, ${ }^{3} \mathrm{H}-\mathrm{CEt}$ labeled HDL $\left(5 \times 10^{5} \mathrm{dpm}\right),{ }^{125}$ I-tyramine-cellobiose apoA-I-labeled HDL $\left(10^{5} \mathrm{dpm}\right)$, or ${ }^{125} \mathrm{I}$-apoA-I-labeled HDL $\left(10^{5} \mathrm{dpm}\right)$ was injected into the saphenous veins of mice. The plasma decay curves and the fractional catabolic rates (FCRs) were generated using a multiexponential curve fitting technique on the WINSAAM program (National Cancer Institute, NIH, Bethesda, Maryland, USA) (29).

Five hours after radiotracer injection, mice $(n=4$ each group) were euthanized and liver, kidneys, testis, small intestine, heart, adrenals and spleen were harvested. ${ }^{3} \mathrm{CEt}$ was extracted in 20 volumes of chloroformmethanol, $2: 1$ (vol/vol). ${ }^{3} \mathrm{CEt}$ and ${ }^{125}$ I were counted in the different organs. Mean recoveries of organs and plasma were higher than $80 \%$ of injected dose. The uptake of both tracers by murine tissues was expressed in terms of organ FCRs calculated by multiplying the plasma FCR of a given radiotracer with the amount of the tracer recovered in that organ divided by the total radioactivity recovered from all extravascular sources. The absolute delivery of a specific radiotracer ${ }^{125} \mathrm{I}$ or ${ }^{3} \mathrm{CEt}$ ) to various tissues equaled the product of the organ FCR and the plasma pool size $(32,33)$.

$V L D L$ apoB production. To determine apoB secretion control $(n=5)$ and hABCA1-A Tg $(n=5)$, mice were injected with Triton WR 1339 (500 mg/kg; Sigma Chemical Co., St. Louis, Missouri, USA) via the saphenous vein to block lipolysis. Five minutes later, mice were injected with $100 \mu \mathrm{Ci}$ of $\left[{ }^{35} \mathrm{~S}\right]$ methionine (New England Nuclear, Boston, Massachusetts, USA) to label newly synthesized protein. Mice were bled at 0,90 , and 180 minutes after Triton injection. Plasma VLDL was isolated by ultracentrifugation $(d=1.006$, top) and analyzed by polyacrylamide gradient gel electrophoresis (3-12\%; Invitrogen 


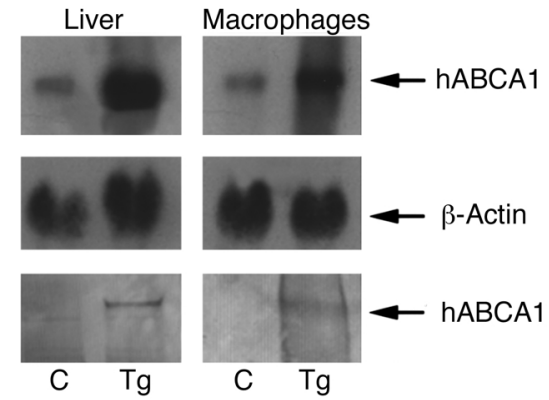

b

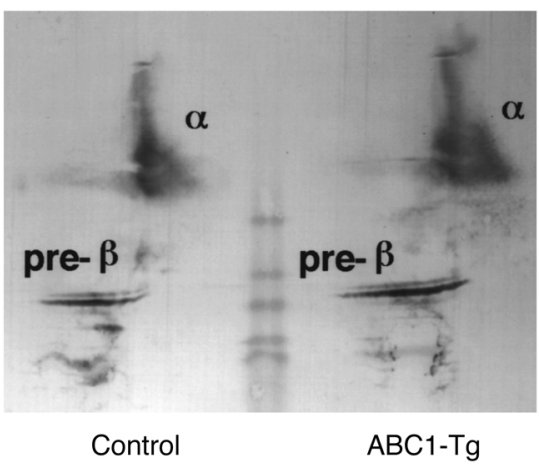

c

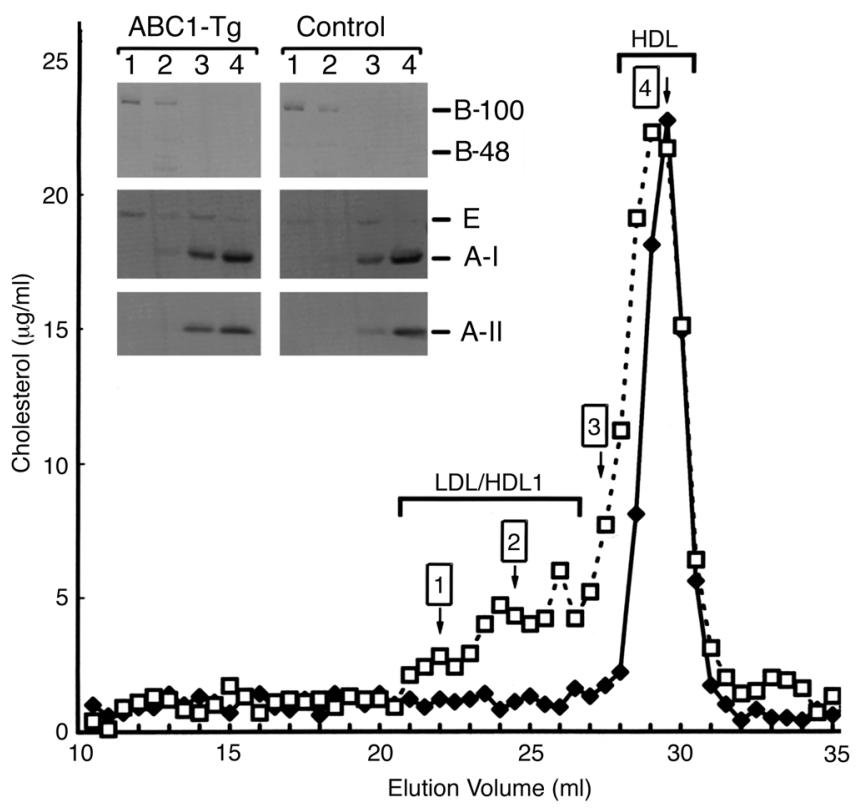

Figure 1

Hepatic and macrophage expression of human ABCA1 and plasma lipoprotein analysis in control and hABCA1-Tg mice. (a) Northern blot analysis of total RNA $(20 \mu \mathrm{g})$ from representative control $(C)$ and hABCA1-A Tg male mice. Human ABCA1 and $\beta$-actin cDNAs were utilized as probes (top and middle). Immunoblot analysis of mouse liver and macrophage homogenates ( $25 \mu \mathrm{g}$ each) was performed using an antihuman ABCA1 polyclonal antibody (bottom). (b) Two-dimensional gel electrophoresis analysis of $15 \mu$ l of pooled plasma $(n=5$; each group) from control and hABCA1-A Tg mice. (c) FPLC elution profile of pooled plasma $(60 \mu \mathrm{l})$ from control (filled diamonds) and hABCA1-A Tg (open squares) mice. Ten microliters of FPLC fractions 1, 2, 3, and 4 was analyzed by immunoblotting as described in Methods (c, inset).

Corp., Carlsbad, California, USA) followed by immunoblotting using anti-apoB antibodies from Biodesign International. Alternatively, the apoB-100 and apoB-48 gel bands were isolated and counted in CytoScint (ICN Radiochemicals, Costa Mesa, California, USA), or the polyacrylamide gel was dried and exposed in the PhosphorImager 445SI (Molecular Dynamics) to quantify the $\left.{ }^{35} \mathrm{~S}\right]$ methionine incorporated into apoB-100 and apoB-48. The mean apoB value for control mice $(n=5)$ was calculated, and the apoB value of each hABCA1-A Tg mice $(n=5)$ was expressed as a percentage relative to the mean apoB value for control mice. Bile acid quantification. Mouse gallbladders excised at necropsy were spun, and the bile was collected and stored frozen. Biliary cholesterol, bile acids (Sigma Diagnostics kits 352 and 450-A; Sigma Chemical Co.), and phospholipids (Wako Commercial kit 996-54001; Wako Chemicals Inc., Richmond, Virginia, USA) were measured according to the manufacturers' instructions.

Statistical analysis. Values are reported as mean \pm SEM. Comparisons between control and transgenic mice were made using the Student's $t$ test for independent samples (two-tailed) and ANOVA.

\section{Results}

ABCA1 expression and cholesterol efflux in ABCA1-Tg mice. Two separate lines of ABCA1-Tg mice containing 30 (hABCA1-A) and 40 (hABCA1-B) copies of the human
ABCA1 gene were established. Northern blot hybridization analysis and densitometric scanning demonstrated increased hABCA1 mRNA in the liver (four- and ninefold, respectively) and macrophages (three- and sixfold, respectively) of hABCA1-A Tg mice (Figure 1a, top and middle) and hABCA1-B Tg mice (data not shown). Human ABCA1 mRNA expression was not increased in brain, adrenals, heart, small intestine, spleen, lung, or kidney. The human ABCA1 250$\mathrm{kDa}$ protein was identified in both mouse liver and macrophage cell extracts isolated from hABCA1-Tg but not control mice (Figure 1a, bottom) by Western blotting using anti-human ABCA1 polyclonal antibodies. ABCA1 expression enhanced apoA-I cholesterol efflux in peritoneal macrophages approximately twofold $(P<0.01)$ in hABCA1-Tg $(21 \pm 3 \%)$ and compared with controls $(10 \pm 1 \%)$.

Plasma lipids, lipoproteins, and apolipoproteins in ABCA1$\mathrm{Tg}$ mice. Male and female ABCA1-Tg mice from both lines (Table 1) had elevated fasting plasma TC, PL, FC, $\mathrm{CE}$, and HDL-C compared with controls. The plasma lipids for hABCA1-B $\mathrm{Tg}$ mice were intermediate between controls and hABCA1-A Tg mice, indicating a gene-related dose response. ApoA-I and apoB levels were increased approximately 1.3 -fold in male and female hABCA1-A Tg mice, but TG and apoE levels were elevated only in male hABCA1-A Tg mice (Table 1). Although non-HDL-cholesterol (apoB-containing 
Table 1

Plasma lipids, lipoproteins, and apolipoproteins in human ABCA1 transgenic and control mice

\begin{tabular}{|c|c|c|c|c|c|c|c|c|c|c|c|}
\hline Genotype & $\mathrm{TC}$ & TG & $\mathrm{PL}$ & FC & CE & $\begin{array}{l}\text { HDL-C } \\
(\mathrm{mg} / \mathrm{dl})\end{array}$ & Non-HDL-C & ApoA-I & ApoA-II & ApoE & ApoB \\
\hline \multicolumn{12}{|l|}{ Females } \\
\hline $\begin{array}{l}\text { hABCA1-A Tg } \\
(n=6)\end{array}$ & $127 \pm 7^{B}$ & $54 \pm 10$ & $192 \pm 12^{\mathrm{B}}$ & $24 \pm 1^{B}$ & $103 \pm 7^{B}$ & $117 \pm 7^{B}$ & $10 \pm 4$ & $112 \pm 4^{C}$ & $37 \pm 2^{B}$ & $3 \pm 1$ & $36 \pm 3^{A}$ \\
\hline $\begin{array}{l}\text { hABCA1-B Tg } \\
(n=7)\end{array}$ & $100 \pm 6^{B}$ & $67 \pm 5$ & $150 \pm 10^{\mathrm{B}}$ & $19 \pm 2^{B}$ & $81 \pm 5^{B}$ & $83 \pm 7^{B}$ & $17 \pm 9$ & $86 \pm 6$ & $34 \pm 3$ & $3 \pm 1$ & $34 \pm 3$ \\
\hline $\begin{array}{l}\text { Controls } \\
(n=12)\end{array}$ & $76 \pm 2$ & $61 \pm 5$ & $131 \pm 6$ & $13 \pm 1$ & $63 \pm 2$ & $70 \pm 2$ & $6 \pm 3$ & $84 \pm 3$ & $27 \pm 1$ & $3 \pm 1$ & $27 \pm 1$ \\
\hline \multicolumn{12}{|l|}{ Males } \\
\hline $\begin{array}{l}\text { hABCA1-A Tg } \\
(n=9)\end{array}$ & $187 \pm 10^{C}$ & $89 \pm 3^{c}$ & $285 \pm 13^{c}$ & $40 \pm 2^{C}$ & $147 \pm 9 c$ & $163 \pm 7 c$ & $24 \pm 8$ & $100 \pm 6^{A}$ & $37 \pm 2$ & $5 \pm 1^{\mathrm{A}}$ & $39 \pm 2^{B}$ \\
\hline $\begin{array}{l}\text { hABCA1-B Tg } \\
(n=9)\end{array}$ & $158 \pm 8^{C}$ & $83 \pm 8^{c}$ & $253 \pm 7^{c}$ & $32 \pm 2^{c}$ & $126 \pm 7^{c}$ & $125 \pm 6^{C}$ & $33 \pm 13$ & $110 \pm 16$ & $38 \pm 5$ & $4 \pm 1$ & $36 \pm 2^{A}$ \\
\hline $\begin{array}{l}\text { Controls } \\
(n=18)\end{array}$ & $97 \pm 4$ & $68 \pm 5$ & $189 \pm 7$ & $19 \pm 1$ & $79 \pm 3$ & $81 \pm 4$ & $16 \pm 9$ & $83 \pm 4$ & $34 \pm 3$ & $2 \pm 0$ & $28 \pm 1$ \\
\hline
\end{tabular}

lipoprotein cholesterol) appeared to be increased in hABCA1-Tg mice, the levels were not significantly different between control and transgenic male and female mice on a regular chow diet (Table 1).

Increased $\alpha$ - and pre- $\beta$ migrating lipoproteins were detected in the plasma of hABCA1-Tg mice by twodimensional gel electrophoresis followed by immunoblotting with anti-human apoA-I antibody (Figure 1b). FPLC analysis of the plasma lipoproteins revealed that the majority of the cholesterol was associated with fractions corresponding to HDL1/HDL in ABCA1-Tg mice (Figure 1c). A small increase in the cholesterol associated with the apoB-100-containing LDL fraction was also noted. Increased apoE in LDL, HDL1, and HDL hABCA1-Tg mice FPLC fractions was revealed by immunoblotting (Figure 1c, inset). HDL from ABCA1-Tg mice showed a broader peak with a slight shift to the left consistent with cholesterol enrichment.

HDL metabolism. To investigate the effect of ABCA1 overexpression on plasma HDL metabolism, we performed HDL kinetic studies using nondegradable as well as degradable radiolabels $(30,32)$. The plasma decay of ${ }^{125}$ I-apoA-I HDL (Figure 2, top) was significantly delayed in ABCA1-Tg mice compared with controls with FCRs of $1.42 \pm 0.12 \mathrm{~d}^{-1}$ vs. $2.17 \pm 0.09 \mathrm{~d}^{-1}$, respectively. Thus, delayed catabolism of apoA-I HDL led to increased plasma apoA-I levels in hABCA-1 Tg mice. Although a similar trend was observed for ${ }^{3} \mathrm{H}-\mathrm{CEt}$ HDL kinetics (Figure 2, bottom), the difference in the FCR between control $\left(3.09 \pm 0.25 \mathrm{~d}^{-1}\right)$ and hABCA1-Tg $\left(2.02 \pm 0.32 \mathrm{~d}^{-1}\right)$ mice did not reach statistical significance $(P=0.09)$. These data indicate that the increase HDL-CE in hABCA1-Tg mice is not due to delayed HDL-CEt clearance. Instead, as discussed previously, the increase plasma HDL-CE pool size may result at least in part, from enhanced ABCA-1-mediated cellular cholesterol efflux to apoA-I HDL.

Five hours after injection, a significant difference $(P<$ 0.009 ; all $)$ in the amount of ${ }^{125} \mathrm{I}$-tyramine cellobiose labeled apoA-I present in the hABCA1-Tg versus control mouse plasma ( $51.8 \pm 1.5 \%$ vs. $40.7 \pm 1.2 \%)$, kidney $(15.6$ $\pm 0.2 \%$ vs. $19.8 \pm 0.6 \%)$, and liver $(28.8 \pm 0.7 \%$ vs. $35.4 \pm$ $0.7 \%$ ) was noted. However, the absolute delivery of HDL-apoA-I to the liver, which is dependent on the HDL-apoA-I plasma pool size was similar in both animal groups (control $=1.1 \pm 0.3 \mathrm{mg}$ apoA- $\mathrm{I} / \mathrm{d}$ versus hABCA1-Tg $=1.2 \pm 0.2 ; P>0.05)$. In contrast, the HDL ${ }^{3} \mathrm{H}-\mathrm{CEt}$ content of hABCA1-Tg versus control mouse plasma $(26.6 \pm 2.7 \%$ vs. $19.7 \pm 3.0 \%$ and liver $(48.8 \pm 1.7 \%$ vs. $55.9 \pm 3.1 \%$ was similar $(P>0.05$; all $)$. However, the absolute delivery of HDL-CEt to the liver, which is dependent on HDL-CE pool size, was increased 1.5-fold $(P<0.001)$ in hABCA1-Tg $(2.41 \pm 0.09 \mathrm{mg} \mathrm{CE} / \mathrm{d})$ versus control mice $(1.66 \pm 0.09 \mathrm{mg} \mathrm{CE} / \mathrm{d})$. These data indicate that the hepatic delivery of CE per HDL particle is greater in hABCA1-Tg mice due to CE enrichment.

$V L D L-a p o B$ production. To evaluate potential mechanisms leading to increased plasma apoB in hABCA1-A $\mathrm{Tg}$ mice, we injected Triton WR1339 and ${ }^{35} \mathrm{~S}$-methionine in mice. Immunoblot analysis of VLDL-apoB in control and hABCA1-A Tg mice 1.5 hours (data not shown) and 3 hours (Figure 3 ) after Triton infusion demonstrated no significant differences in apoB-100 and apoB-48 levels. These findings were also confirmed by gel quantitation of ${ }^{35} \mathrm{~S}$-methionine incorporated into VLDL-apoB 3 hours after Triton infusion as described in Methods. The incorporation of ${ }^{35} \mathrm{~S}$-methionine into apoB-100 and apoB-48 hABCA1-A Tg mice $(n=5)$ was similar to that of control mice $(n=5)$. These data demonstrate that the increased plasma apoB in hABCA1-Tg mice is not due to increased apoB secretion. 


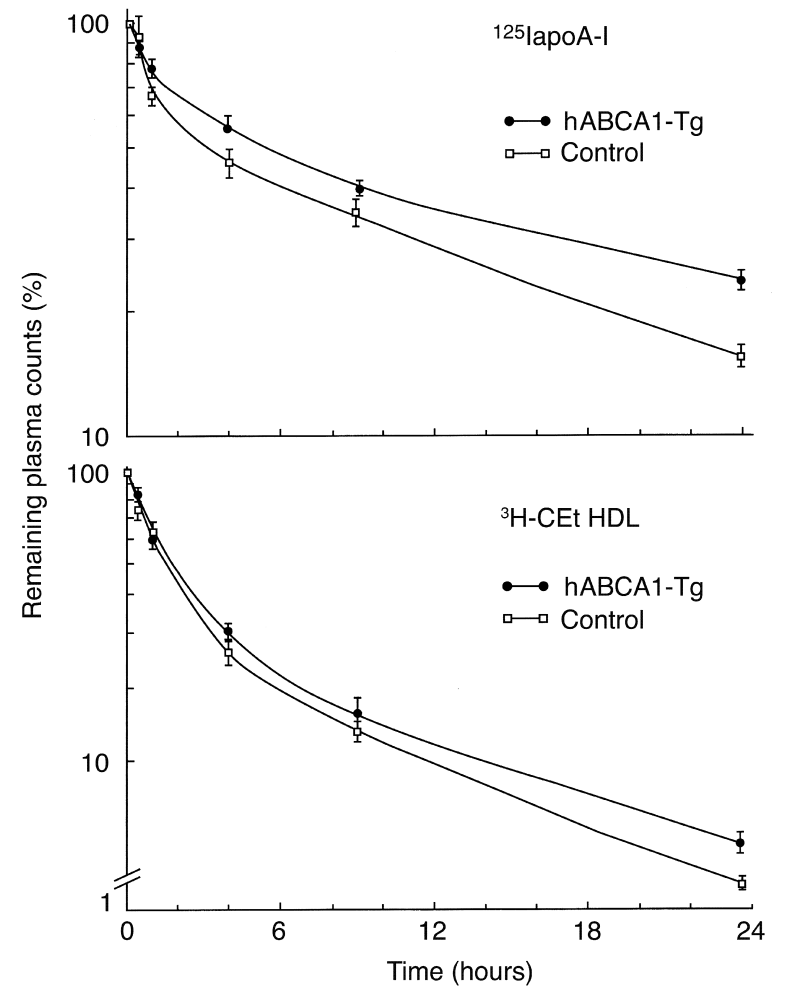

Figure 2

Kinetic analysis of apoA-I and CEt HDL catabolism. ${ }^{125}$ I apoA-I and ${ }^{3} \mathrm{H}$-CEt HDLs were injected into male hABCA1-B Tg and C57BL/ 6 mice ( $n=4$ mice per group). Values indicate the percent remaining counts in plasma compared with the 1-minute value expressed as mean \pm SEM. The FCRs were determined from the area under the curve using an exponential curve fitting technique on the WINSAAM program using data points through 24 hours of study. ApoA-I HDL FCRs calculated using data points through 48 hours of study were similar (controls $=2.0 \pm 0.1 \mathrm{~d}^{-1}$ and hABCA1-Tg $\left.=1.30 \pm 0.04 \mathrm{~d}^{-1} ; P<0.01\right)$.

Bile composition. Overexpression of hABCA1 led to an increase $(P<0.05$; all $)$ in the cholesterol and PL content of bile in hABCA1-B Tg $(n=5$; C: $137.0 \pm 14.0$ $\mathrm{mg} / \mathrm{dl}$, and PL: $15.1 \pm 1.5 \mathrm{~g} / \mathrm{l})$ compared with controls $(n=9 ; \mathrm{C}: 76.2 \pm 7.5 \mathrm{mg} / \mathrm{dl}$, and PL: $9.0 \pm 0.7 \mathrm{~g} / \mathrm{l})$. This represented an approximately 1.8 -fold increase in the concentration of both lipids in the bile of hABCA1$\mathrm{Tg}$ mice. In contrast, the bile acid content was not altered (hABCA1-B Tg: $58.8 \pm 7.3 \mathrm{mM}$ and controls: $48.2 \pm 7.1 \mathrm{mM})$. Northern blot hybridization analysis of mdr2, a major transporter that facilitates bile phospholipid excretion (34), revealed that hepatic mdr2 mRNA was not increased in hABCA1-Tg mice (data not shown).

\section{Discussion}

To investigate the consequences of enhanced ABCA1 expression on HDL metabolism, reverse cholesterol transport, and hepatic biliary excretion in vivo, we produced two independent lines of transgenic mice, which overexpress human ABCA1 under control of the apoE promoter. This promoter targeted expression of the human transgene primarily to the liver and macrophages of hABCA1-Tg mice, two tissues where ABCA1 is normally expressed (10). Previous studies $(12,14,20)$ have indicated an important function for ABCA1 in the removal of cholesterol from macrophages. Foam cells, resulting from the accumulation of excess cholesterol in macrophages, lead to the formation of fatty streaks, complex lesions, and eventually plaque rupture (35). Stimulation of macrophage cholesterol efflux by enhanced ABCA1 expression may inhibit foam cell formation and consequently reduce atherogenic risk. In contrast, the role of ABCA1 in hepatic cholesterol homeostasis is less clearly understood. Depending on its location, ABCA1 may enhance hepatic cholesterol efflux into plasma lipoproteins, facilitate biliary cholesterol excretion, or both. These two potential ABCA1-mediated hepatic processes could have opposite consequences on reverse cholesterol transport.

The present study establishes that enhanced ABCA1 expression in liver and macrophages has a significant impact on the plasma lipid profile, leading to elevated plasma cholesterol, HDL cholesterol, and apoA-I in a gene-related dose-response fashion. The HDL in hABCA1-Tg mice is cholesterol enriched, and apoE-rich HDL1 accumulates. Increased HDL1 has been previously reported in mice with SR-BI deficiency $(36,37)$ and LCAT overexpression (29), metabolic conditions under which the cholesterol content of HDL is increased by either reduced HDL cholesterol selective uptake or enhanced HDL-C esterification. Interestingly, pre- $\beta$ HDL, the subfraction of HDL, which is very effective in cellular cholesterol efflux (2), were increased in hABCA1-Tg mice.

Cavelier et al. (38) recently reported the generation of two different transgenic mouse lines from BACs containing the hABCA1 gene. In contrast to our find-

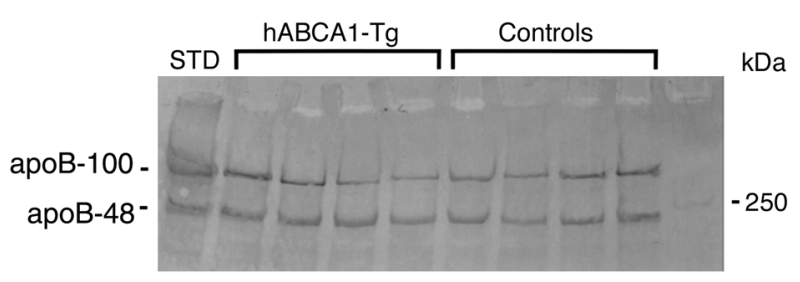

\section{Figure 3}

VLDL-apoB production after Triton WR1399 infusion. Control $(n=$ $5)$ and hABCA1-A Tg $(n=5)$ mice were injected with Triton WR1399 to block lipolysis and ${ }^{35} \mathrm{~S}$-methionine to label newly synthesized proteins. The mice were bled 3 hours after Triton infusion and VLDL isolated by ultracentrifugation $(d=1.006)$ was analyzed by either immunoblotting with anti-apoB antibodies or quantification of ${ }^{35} \mathrm{~S}$ methionine incorporation into apoB-100 and apoB-48 (see below). The mean apoB value for control mice $(n=5)$ was calculated. The value of hABCA1-A Tg mice $(n=5)$ expressed as a percentage relative to the mean apoB value for control mice was: for apoB-100, 95 $\pm 11 \%$, and for apoB-48, $103 \pm 7 \%(P>0.05)$. STD, standard. 
ings, they reported that mice with increased hABCA1 expression in liver (twofold; determined by quantitative RT-PCR) and macrophages (twofold; determined by increase cholesterol efflux) or testis had no changes in the plasma lipids or in HDL-C levels. In the present study, hABCA1 expression was increased four- to ninefold in liver and three- and sixfold in macrophages in hABCA1-Tg mice. Thus, lower hABCA1 expression levels may account for the lack of increased plasma HDL concentrations in transgenic mice generated using hABCA1 BACs (38). These authors have suggested (38) that interaction between human ABCA1 and endogenous mouse ligands may be inefficient requiring higher levels of expression to alter the plasma lipid profile. Differences in in vivo function of LCAT have been reported in transgenic mice overexpressing the mouse versus the human enzyme $(29,39)$.

Kinetic studies using degradable and nondegradable protein labels in hABCA1-Tg showed that ABCA1 overexpression increased plasma apoA-I HDL levels by reducing the catabolism of apoA-I in both the liver and kidney of transgenic mice. Although the pool size of apoA-I HDL in hABCA1-Tg mice is larger than control mice, differences in HDL pool size have been previously shown not to have a major effect on FCR in apoA-I $\mathrm{KO}$ mice (33), in patients with Tangier disease before and after HDL infusion (40) or in a patient with marked hyperalphalipoproteinemia (41). Several studies have demonstrated that one of the major determinants of HDL catabolism is HDL size (42). Larger, lipid-rich HDL appears to be catabolized more slowly (42). Lipid enrichment of HDL may alter the conformation and accessibility of surface apolipoproteins for interaction with cell-surface receptors responsible for HDL catabolism (43). The enhanced lipidation of HDL facilitated by ABCA1-mediated cellular efflux in hABCA1-Tg mice, which leads to the formation of larger, CE-enriched HDL, may delay hABCA1-Tg HDL catabolism by a similar mechanism.

The metabolism of ${ }^{3} \mathrm{H}-\mathrm{CEt} \mathrm{HDL}$ was also altered by ABCA1 expression. Although the plasma and liver FCRs of ${ }^{3} \mathrm{H}$-CEt HDL were similar in both study groups, when the increased plasma HDL-CE pool size was taken into account the absolute rate of hepatic HDL-CE delivery was increased by $750 \mu \mathrm{g} / \mathrm{d}$ in hABCA1-Tg, an amount equal to the entire CE pool size of control mice. These data indicate that increased ABCA1 expression in both macrophages and liver enhances the net hepatic delivery of exogenous HDL$\mathrm{CE}$ in vivo. Of major interest is the finding that the cholesterol and PL content of bile was increased approximately 1.8 -fold. Mdr2 deficiency in mice leads to defective biliary cholesterol and PL excretion (34, 44); however, $\mathrm{mdr} 2$ gene expression was unaltered in hABCA1-Tg mice. Increases in bile cholesterol but not $\mathrm{PL}$ content have been reported in mice overexpressing SR-BI $(45,46)$ and hABCG1 (47). Our findings indicate that hepatic ABCA1 overexpression may directly facilitate the transport of both cholesterol and PL into bile.
Further studies will be required to identify potential mechanisms by which ABCA1 overexpression modulates biliary lipid secretion. The lack of concomitant changes in bile acid concentrations in these three mouse models indicates independent regulation of the $17-\alpha$ hydroxylase bile acid and cholesterol excretion pathways in bile.

The combined metabolic changes that result from ABCA1 overexpression reported in this study would be predicted to reduce atherogenic risk in hABCA1-Tg mice. In contrast, increased plasma apoB levels in hABCA1 Tg mice, should counter these potentially beneficial effects of ABCA1 overexpression. Decreased plasma apoB levels has been described in ABCA1knockout mice (22-24), but the underlying mechanism has not been reported. Here we demonstrate that the increase in plasma apoB levels in hABCA1-Tg mice is not due to increased apoB secretion, consistent with delayed catabolism.

Our combined studies show, for the first time to our knowledge, that $\mathrm{ABCA} 1$ overexpression in macrophages and liver alters the plasma lipid profile and modulates plasma HDL catabolism, hepatic reverse cholesterol transport, and biliary cholesterol excretion in vivo and thus may markedly alter atherogenic risk in humans.

\section{Acknowledgments}

We thank John M. Taylor for the gift of the pLIV.11 construct and Donna James for excellent secretarial assistance.

1. Miller, N.E. 1987. Associations of high-density lipoprotein subclasses and apolipoproteins with ischemic heart disease and coronary atherosclerosis. Am. Heart J. 113:589-597.

2. Fielding, C.J., and Fielding, P.E. 1997. Intracellular cholesterol transport. J. Lipid Res. 38:1503-1521.

3. Oram, J.F., and Yokoyama, S. 1996. Apolipoprotein-mediated removal of cellular cholesterol and phospholipids. J. Lipid Res. 37:2473-2491.

4. Dean, M., and Allikmets, R. 1995. Evolution of ATP-binding cassette transporter genes. Curr. Opin. Genet. Dev. 5:779-785.

5. Decottignies, A., and Goffeau, A. 1997. Complete inventory of the yeast ABC proteins. Nat. Genet. 15:137-145.

6. Remaley, A.T., et al. 1999. Human ATP-binding cassette transporter 1 (ABC1): genomic organization and identification of the genetic defect in the original Tangier disease kindred. Proc. Natl. Acad. Sci. USA. 96:12685-12690.

7. Santamarina-Fojo, S., et al. 2000. Complete genomic sequence of the human ABCA1 gene: analysis of the human and mouse ATP-binding cassette A promoter. Proc. Natl. Acad. Sci. USA. 97:7987-7992.

8. Brousseau, M.E., et al. 2000. Novel mutations in the gene encoding ATPbinding cassette 1 in four tangier disease kindreds. J. Lipid Res. 41:433-441.

9. Pullinger, C.R., et al. 2000. Analysis of hABC1 gene $5^{\prime}$ end: additional peptide sequence, promoter region, and four polymorphisms. Biochem. Biophys. Res. Commun. 271:451-455.

10. Langmann, T., et al. 1999. Molecular cloning of the human ATP-binding cassette transport 1 (hABC1): evidence of sterol-dependent regulation in macrophages. Biochem. Biophys. Res. Commun. 257:29-33.

11. Lawn, R.M., et al. 1999. The Tangier disease gene product ABC1 controls the cellular apolipoprotein-mediated lipid removal pathway. J. Clin. Invest. 104:R25-R31.

12. Chen, W., Silver, D.L., Smith, J.D., and Tall, A.R. 2000. Scavenger receptor-BI inhibits ATP-binding cassette transporter 1-mediated cholesterol efflux in macrophages. J. Biol. Chem. 275:30794-30800.

13. Takahashi, Y., et al. 2000. Identification of cAMP analogue inducible genes in RAW264 macrophages. Biochim. Biophys. Acta. 1492:385-394.

14. Sakr, S.W., Williams, D.L., Stoudt, G.W., Phillips, M.C., and Rothblat, G.H. 1999. Induction of cellular cholesterol efflux to lipid-free apolipoprotein A-I by cAMP. Biochim. Biophys. Acta. 1438:85-98.

15. Rust, S., et al. 1999. Tangier disease is caused by mutations in the ATP 
binding cassette transporter 1 (ABC1) gene. Nat. Genet. 22:352-355.

16. Brooks-Wilson, A., et al. 1999. Mutations in ABC1 in Tangier disease and familial high-density lipoprotein deficiency. Nat. Genet. 22:336-345.

17. Bodzioch, M., et al. 1999. The gene encoding ATP-binding cassette transporter 1 is mutated in Tangier disease. Nat. Genet. 22:347-351.

18. Wang, J., et al. 2000. Common and rare ABCA1 variants affecting plasma HDL cholesterol. Arterioscler. Thromb. Vasc. Biol. 20:1983-1989.

19. Mott, S., et al. 2000. Decreased cellular cholesterol efflux is a common cause of familial hypoalphalipoproteinemia: role of the ABCA1 gene mutations. Atherosclerosis. 152:457-468.

20. Oram, J.F., and Vaughan, A.M. 2000. ABCA1-mediated transport of cellular cholesterol and phospholipids to HDL apolipoproteins. Curr. Opin. Lipidol. 11:253-260.

21. Remaley, A.T., et al. 1997. Decreased reverse cholesterol transport from Tangier disease fibroblasts. Acceptor specificity and effect of brefeldin on lipid efflux. Arterioscler. Thromb. Vasc. Biol. 17:1813-1821.

22. McNeish, J., et al. 2000. High density lipoprotein deficiency and foam cell accumulation in mice with targeted disruption of ATP-binding cassette transporter-1. Proc. Natl. Acad. Sci. USA. 97:4245-4250.

23. Christiansen-Weber, T.A., et al. 2000. Functional loss of ABCA1 in mice causes severe placental malformation, aberrant lipid distribution, and kidney glomerulonephritis as well as high-density lipoprotein cholesterol deficiency. Am. J. Pathol. 157:1017-1029.

24. Orso, E., et al. 2000. Transport of lipids from golgi to plasma membrane is defective in tangier disease patients and Abc1-deficient mice. Nat. Genet. 24:192-196.

25. Fan, J., et al. 1994. Overexpression of hepatic lipase in transgenic rabbits leads to a marked reduction of plasma high density lipoproteins and intermediate density lipoproteins. Proc. Natl. Acad. Sci. USA. 91:8724-8728.

26. Vaisman, B.L., et al. 1995. Overexpression of human lecithin cholesterol acyltransferase leads to hyperalphalipoproteinemia in transgenic mice. J. Biol. Chem. 270:12269-12275.

27. Sullivan, P.M., et al. 1997. Targeted replacement of the mouse apolipoprotein E gene with the common human APOE3 allele enhances diet-induced hypercholesterolemia and atherosclerosis. J. Biol. Chem. 272:17972-17980.

28. Asztalos, B.F., Sloop, C.H., Wong, L., and Roheim, P.S. 1993. Two-dimensional eletrophoresis of plasma lipoproteins: recognition of new apo AI containing subpopulations. Biochim. Biophys. Acta. 1169:291-300.

29. Berard, A.M., et al. 1997. High plasma HDL concentrations associated with enhanced atherosclerosis in transgenic mice overexpressing lecithin cholesteryl acyltransferase. Nat. Med. 3:744-749.

30. Pittman, R.C., and Taylor, C.A., Jr. 1986. Methods for assessment of tissue sites of lipoprotein degradation. Methods Enzymol. 129:612-628.

31. McFarlane, A.S. 1958. Efficient trace-labeling of proteins with iodine. Nature. 182:53.

32. Rinninger, F., and Pittman, R.C. 1987. Regulation of the selective uptake of high density lipoprotein-associated cholesteryl esters. J. Lipid Res. 28:1313-1325.

33. Plump, A.S., et al. 1997. ApoA-I knockout mice: characterization of HDL metabolism in homozygotes and identification of a post-RNA mechanism of apoA-I up-regulation in heterozygotes. J. Lipid Res. 38:1033-1047.

34. Smit, J.J., et al. 1993. Homozygous disruption of the murine mdr2 P-glycoprotein gene leads to a complete absence of phospholipid from bile and to liver disease. Cell. 75:451-462.

35. Ross, R. 1993. The pathogenesis of atherosclerosis: a perspective for the 1990s. Nature. 362:801-809.

36. Varban, M.L., et al. 1998. Targeted mutation reveals a central role for SR$\mathrm{BI}$ in hepatic selective uptake of high density lipoprotein cholesterol. Proc. Natl. Acad. Sci. USA. 95:4619-4624.

37. Rigotti, A., et al. 1997. A targeted mutation in the murine gene encoding the high density lipoprotein (HDL) receptor scavenger receptor class B type I reveals its key role in HDL metabolism. Proc. Natl. Acad. Sci. USA. 94:12610-12615.

38. Cavelier, L.B., et al. 2001. Regulation and activity of the human ABCA1 gene in transgenic mice. J. Biol. Chem. 21:18046-18051.

39. Amar, M.J.A., et al. 1999. Expression of murine LCAT in transgenic mice: evaluation of the role of LCAT cholesteryl ester-fatty acid specificity in the development of atherosclerosis. Circulation. 100:I-401. (Abstr.)

40. Schaefer, E.J., et al. 1981. Metabolism of high density lipoprotein subfractions and constituents in Tangier disease following the infusion of high density lipoproteins. J. Lipid Res. 22:217-228.

41. Rader, D.J., et al. 1993. Increased production of apolipoprotein A-I associated with elevated plasma levels of high-density lipoproteins, apolipoprotein A-I, and lipoprotein A-I in a patient with familial hyperalphalipoproteinemia. Metabolism. 42:1429-1434.

42. Brinton, E.A., Eisenberg, S., and Breslow, J.L. 1994. Human HDL cholesterol levels are determined by apoA-I fractional catabolic rate, which correlates inversely with estimates of HDL particle size: effects of gender, hepatic and lipoprotein lipases, triglyceride and insulin levels, and body fat distribution. Arterioscler. Thromb. 14:707-720.

43. Lux, S.E., Hirz, R., Shrager, R.I., and Gotto, A.M., Jr. 1972. The influence of lipid on the conformation of human plasma high density apolipoproteins. J. Biol. Chem. 247:2598-2606.

44. van Nieuwerk, C.M., et al. 1997. The role of bile salt composition in liver pathology of mdr2 (-/-) mice: differences between males and females. $J$. Hepatol. 26:138-145.

45. Kozarsky, K.F., et al. 1997. Overexpression of the HDL receptor SR-BI alters plasma HDL and bile cholesterol levels. Nature. 387:414-417.

46. Sehayek, E., et al. 1998. Biliary cholesterol excretion: a novel mechanism that regulates dietary cholesterol absorption. Proc. Natl. Acad. Sci. USA. 95:10194-10199.

47. Ito, T., et al. 2000. Adenovirus-mediated expression establishes an in vivo role for human ABCG1 (ABC8) in lipoprotein metabolism. Circulation. 102:II-311. (Abstr.) 